\title{
Feto papiráceo - presentación de casos
}

\section{Fetus papyraceus - presentation of cases}

Edgar E. Rivas-Perdomo', José Sánchez Reina²

\section{Resumen}

El embarazo gemelar con un feto papiráceo es una entidad rara. Se presentan dos casos de embarazo gemelar con la muerte de uno de los fetos y su posterior transformación a lo conocido como feto papiráceo. El primero de los casos se detectó el óbito de uno de los gemelos a las 29 semanas y nacieron a las 35 ; el sobreviviente no presentó complicaciones. El segundo caso se detectó a las 21 semanas y nacieron a las 34 semanas. En ambos casos bicorial biamniótico, la causa de muerte: hipoxia fetal extrínseca por insuficiencia feto-placentaria.

Palabras clave: Embarazo múltiple, feto, gemelos, muerte fetal.

\begin{abstract}
Twin pregnancy with a fetus papyraceus is rare. Two cases of twin pregnancy with the death ofone fetus and its subsequent transformation to fetus papyraceus are shown. In the first case it was detected the death of one twin at 29 weeks and was born at 35 weeks; the survivor had no complications. The second case was detected at 21 weeksand was bornat 34 weeks. In both cases diamniotic dichorionic, the cause of death was extrinsic fetal hypoxiadue to fetal-placental insufficiency.
\end{abstract}

Keywords: Multiple pregnancy, fetus, twins, fetal death.

\section{Introducción}

El embarazo gemelar constituye un cuadro importante, que linda entre lo fisiológico y lo patológico, por lo que algunos autores consideran el embarazo múltiple en humanos una manifestación de atavismo y constituye genéticamente un fenómeno en retroceso $^{[1]}$; por lo tanto no es exagerado considerar que un embarazo con fetos múltiples es un embarazo complicado con un elevado índice de morbilidad. La muerte anteparto de uno de los gemelos es una rara complicación obstétrica que ocurre en 1:184 nacimientos gemelares; otros autores han encontrado una frecuencia de $3,7 \%{ }^{[2]}$ hasta $6.2 \%$ de todos los embarazos gemelares ${ }^{[3]}$. En muchos de estos casos se recoge el antecedente de hipertensión en el embarazo, como una situación inducida o agravada por las gestaciones múltiples $^{[2]}$, aunque los estudios son contradicto$\operatorname{rios}^{[4,5]}$. Debido a la poca frecuencia de estos eventos; hemos entendido útil para el conocimiento de obstetras y Médicos generales, la presentación de estos casos.

\section{Casos clínicos}

Caso 1 1. Paciente de 16 años de edad primigestante ingresa al servicio de urgencias con imágenes diagnosticas (IDx) de: 1) Embarazo gemelar bicorial- biamniótico de 34 semanas con un feto muerto. 2) Trabajo de parto en fase activa. 3) sífilis gestacional. A Personales: Sifilis en el presente embarazo -tratada con penicilina benzatínica 2400000 UI IM-. Hemoclasificación: Grupo B; Factor Rh:+. Ecografía previa de un mes antes indica embarazo gemelar bicorial biamniótico con un feto vivo cuya biometria corresponde a 29 semanas +2 días y un feto sin actividad cardiaca con biometría aproximada para 18 semanas. Se solicitaron estudios. $\mathrm{Hb}: 13.1 \mathrm{~g} / \mathrm{dl}$; Hto: 39,3\%; Leucocitos: 13100/ml; PMN: 81,8\% Linf: $12,9 \%$ Monocitos: 5,3\%. VDRL: Reactivo 8 dil. Anticuerpos VIH 1 y 2: Negativos. Fibrinógeno: $175 \mathrm{mg} / \mathrm{dl}$; TP: 13,3 seg (Control: 13,0 seg); TPT: 28,5 seg (Control: 30,1 seg). FTA-abs: Positivo. Se realizó cesárea. Se obtuvo un recién nacido vivo de sexo masculino, de $1610 \mathrm{~g}$; talla: $45 \mathrm{~cm}$; Apgar de 8 al minuto y 9 a los 5 minutos. Se consideró recién nacido pretérmino, pequeño para la edad gestacional (RNP-PEG). Feto 2: Obitado, aspecto papiráceo, reducido, amorfo. Placenta amorfa. El feto vivo fue atendido en Unidad de Cuidados Intensivos Neonatales durante 20 días, siendo dado de alta.

Patología. Autopsia fetal: Cadáver de feto en formol, sexo masculino con deshidratación importante de sus tejidos. Se observa pequeño y plano (papiráceo). Peso: $140 \mathrm{~g}$; longitud coronilla-talón: $26 \mathrm{~cm}$. Conclusión: Feto de +20 semanas de edad gestacional por biometría fetal macerado, papiráceo. Con severa restricción del

1 MD, MSc. Departamento de Ginecología y Obstetricia. Universidad de Cartagena- Colombia

2 MD. Ginecólogo y Obstetricia. Clínica Madre Bernarda - Cartagena.

Correspondencia: Edgar E. Rivas-Perdomo, erivasp@unicartagena.edu.co

Conflicto de intereses: Ninguno que declarar.

Financiación: Recursos propios de los autores. Estudios de laboratorios realizados durante sus estancias hospitalarias.

Recibido: 23/09/2014 - Revisado: 30/11/2014 - Aceptado: 27/12/2014 
crecimiento intrauterino. Infarto placentario extenso. Membranas y cordón morfológicamente normales. Causa de muerte: hipoxia fetal extrínseca por insuficiencia feto-placentaria.

Caso 2. Paciente de 20 años de edad. Primigestante con embarazo gemelar bicorial biamniótico acude al servicio de urgencias a las 35 semanas de gestación con trabajo de parto en período expulsivo. Refiere historia de un feto muerto, detectado a las 21 semanas de gestación. Se recibió recién nacido vivo femenino de $2480 \mathrm{~g}$ y talla de $48 \mathrm{~cm}$. Apgar al minuto de 8.

Feto 2. Obitado, aspecto papiráceo (Figura 1).

Patología: Cadáver de feto en formol de sexo masculino, con deshidratación importante de sus tejidos, se observa pequeño y plano (Papiráceo). Sin malformaciones fenotípicas. PESO: 40gr, longitud coronilla-talón: $24 \mathrm{~cm}$.

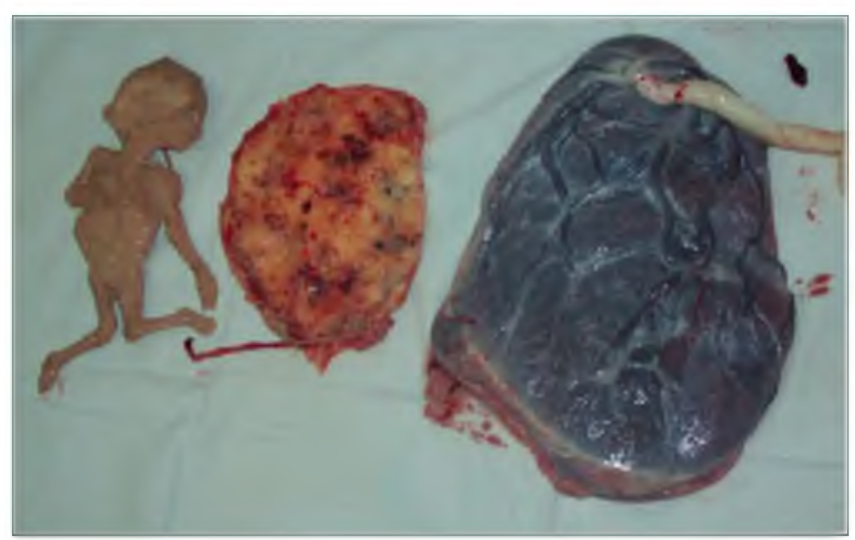

Figura 1. Feto papiráceo (caso 2). Se presenta la placenta del mismo y la placenta del feto sobreviviente.

Conclusión: Feto de +/- 14 semanas de edad gestacional por biometría fetal de sexo masculino, macerado, papiráceo. Feto con severa restricción del crecimiento intrauterino. Infarto placentario extenso.

Causa de muerte: hipoxia fetal extrínseca por insuficiencia feto-placentaria.

\section{Discusión}

Se presentan dos casos de muerte fetal que ocurrieron en la segunda mitad del embarazo generando lo que se conoce como feto papiráceo. E1 término feto papiráceo se utiliza para describir un feto aplanado con pérdida de líquidos y tejidos blandos, asociado con una gestación gemelar o múltiple. La muerte intrauterina de un feto mayor a ocho semanas de gestación y su retención por un mínimo de 10 semanas, da como resultado una compresión mecánica por el feto vivo en crecimiento, con reabsorción del líquido amniótico y del liquido del feto muerto haciendo que asemeje un papel ${ }^{[6,7]}$; dado que por la presencia de huesos y cartílagos no puede ser reabsorbido ${ }^{[8]}$. Se destaca que la compresión parece ser necesaria para que ocurra el fenómeno ${ }^{[9]}$. Está asociado a un tiempo prolongado (pero no estimado) entre la muerte de un gemelo y el parto del sobreviviente ${ }^{[10]}$. La incidencia reportada es de 1:12,000 nacimientos vivos, presentándose 1:184 a 1:200 en embarazos gemelares.
Este fenómeno se asocia más frecuentemente con embarazos gemelares monocoriónico monoamniótico, Russo lo encuentra más frecuentemente en embarazos monocorial diamniótico que en dicorial diamniótico ${ }^{[11]}$; sin embargo, ambos casos fueron bicorialbiamnióticos y ello se muestra de acuerdo con Vargas- Hernández que no es un hallazgo exclusivo de este tipo de embarazos, ya que se han reportado casos con placentación diamniótica-dicoriónica y en muerte fetal por trauma de un gemelo in útero ${ }^{[6]}$. La muerte de un feto en el embarazo gemelar monocoriónico se estima en $12 \%$, mientras en el embarazo dicorónico en solo en $4 \%{ }^{[7]}$, lo que representa un riesgo 3 veces mayor en el embarazo monocorial respecto al bicorial $^{[11]}$.

Se considera que la muerte de un gemelo in útero aumenta el riesgo de morbilidad y mortalidad del gemelo vivo, por la posibilidad de desencadenar la coagulación en la madre, por lo que un control prenatal temprano adecuado y la asistencia médica son necesarios en cualquier embarazo múltiple ${ }^{[6]}$. Ambos partos fueron pretérminos, y un feto con restricción el crecimiento intrauterino lo cual constituye unas de las complicaciones de la muerte de un gemelar $^{[7]}$. Aproximadamente el $57 \%$ de los embarazos gemelares paren antes de las 37 semanas de gestación, aunque no todos los partos pretérminos son espontáneos. Y ello podría ser consecuencia de la liberación de citoquinas y prostaglandinas por la reabsorción de la placenta muerta ${ }^{[12]}$.

Otras complicaciones del feto vivo incluyen presentación o situación distócica, y muerte; se asocian anomalías congénitas como atresia intestinal, gastrosquisis, aplasia cutis, daño al sistema nervioso central ${ }^{[6]}$. Pharoah encuentra un incremento de 5 veces la probabilidad de parálisis cerebral en el feto sobreviviente ${ }^{[13]}$ y cardiopatías, posiblemente atribuibles a los émbolos y a factores que afectan la coagulación ${ }^{[6]}$, aunque esto último es extremadamente raro ${ }^{[7]}$. Con el diagnóstico de muerte fetal in útero, en el curso de un embarazo gemelar es necesario descartar malformaciones en el feto vivo, valorar su curva de crecimiento fetal, volumen de líquido amniótico y madurez placentaria y efectuar perfiles biofísicos seriados. Durante el control prenatal se vigila la coagulación de la madre para descartar una coagulopatía de consumo. El ultrasonido Doppler para la valoración de la circulación umbilical fetal ayuda al diagnóstico del síndrome de transfusión intergemelar y tomar las medidas preventivas ${ }^{[6]}$.

La causa de muerte en ambos casos fue la hipoxia fetal extrínseca por insuficiencia feto-placentaria, secundaria a infarto placentario. Esta es una causa frecuente de muerte fetal y se muestra concordante con lo reportado por otros autores sobre la alta frecuencia de hipoxia fetal extrínseca por patología placentaria como causa de muerte fetal ${ }^{[14,15]}$. Sin embargo, es importante el antecedente de sífilis de la paciente 1 , lo cual es otra causa frecuente de muerte fetal. Otro factor de riesgo que se ha invocado es la edad materna. En el caso uno la paciente tiene una edad de 16 años y en el caso 2 la paciente tiene 20 años, y los estudios son contradictorios. Villalobos en el 2002 encontró mayor frecuencia entre $\operatorname{los} 21-30$ años $^{[16]}$. Rivas-Perdomo en 2013, no encontró asociación significativamente estadística entre la edad materna y la muerte fetal ${ }^{[17]}$,

El pronóstico para el feto sobreviviente es dependiente de la causa de muerte, edad gestacional, corionicidad y el intervalo de tiempo entre la muerte del primer feto y el parto del segundo ${ }^{[18]}$. E1 feto sobreviviente de un embarazo gemelar bicorial complicado con la muerte de un gemelo, está protegido del daño fetal secundario a elementos tromboembólicos y a hipoperfusión por anemia aguda, 
por tener placentas individuales para cada feto ${ }^{[10]}$. Los embarazos gemelares dicigóticos no comparten circulación y la muerte de un gemelar podria tener poco impacto sobre el segundo ${ }^{[18]}$. El mayor riesgo presente en los sobrevivientes de los embarazos bicoriales deriva de la prematurez ${ }^{[10]}$

\section{Referencias}

1. Heredia B. Muerte fetal En: Rigol O. Obstetricia y Ginecología. La Habana CIP-Edt Ciencias Médicas. 2004:237.

2. Peraza $M$; O’Farrill R; Gómez E. Óbito de un gemelo Rev Cubana Obstet Ginecol 2000;26(2):115-118.

3. Hillman SC; Morris RK; Kilby M. Single twin demise: consequence for survivors. Seminars in Fetal \& Neonatal Medicine. 2010;15:319e326. doi:10.1016/ j.siny.2010.05.004

4. Sibai BM; Hauth J; Caritis $S$; Lindheimer MD; MacPherson $C$; Klebanoff $M$. Hypertensive disorders in twin versus singleton gestations. National Institute of Child Health and Human Development Network of Maternal-Fetal Medicine Units. Am J Obstet Gynecol. 2000; 182(4):938-942.

5. Foo J; Mangos $G$; Brown MA. Characteristics of hypertensive disorders in twin versus singleton pregnancies Pregnancy Hypertension: An International Journal of Women's Cardiovascular Health 2013;3:39. doi.org/10.1016/i.preghy.2012.05.005

6. Vargas -Hernández V; Luján J; Ibarra A; Inclán J; Rascón A; López G. Feto papiráceo: complicación de un embarazo gemelar. Rev Hosp Jua Mex 2005;72(4): 165-167.

7. Siddiqui F; McEwan A. Twins. Obstet. Gynaecol reprod Med. 2007;17(10):289-295.

8. Lorenzana-Cruz J. Feto Papiraceo: Presentación de un caso. Rev Med Hond 2002;70:24-26.
9. Peleg D; Ferber A; Orvieto R; Bar-Hava I; Ben-Rafael Z. Single intrauterine fetal death (fetus papyraceus) due to uterine trauma in a twin pregnancy. Europ J Obstet Gynecol Reprod Biology. 1998;80:175-176.

10. Barba S; Carvajal J. Pautas de manejo del embarazo múltiple complicado con la muerte fetal in útero de un gemelo Rev Chil Obstet Ginecol 2012;77(2):159-168.

11. Russo F; Pozzi E; Pelizzoni F; Todyrenchuk L; Bernasconi $D_{i}$ Cozzolino S. et al. Stillbirths in singletons, dichorionic and monochorionic twins: a comparison of risks and causes. European J. Obstet Gynecol Reprod Biol. 2013;170:131-136. doi.org/10.1016/j. ejogrb.2013. 06.014

12. Rao A; Sairam S; Shehata H. Obstetric complications of twin pregnancies. Best Pract Res Clinical Obstet Gynaecol. 2004;18(4):557-576.

13. Pharoah $P$; Adi $Y$. Consequences of in-utero death in a twin pregnancy. Lancet 2000;355:1597-1602.

14. Rivas-Perdomo E.; Vásquez-Deulofeutt D. Óbito fetal: Hallazgos de patología en una institución de alta complejidad. Cartagena, Colombia, 2010-2011. Rev Colomb Obstet Ginecol 2012;63:376-381.

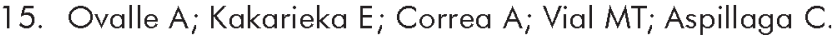
Estudio anátomo-clínico de las causas de muerte fetal. Rev Chil Obstet Ginecol 2005;70(5):303-312.

16. Villalobos $N_{i}$ López C. Síndrome del gemelo muerto. Rev Obstet Ginecol Venez 2002;62(1):11-15.

17. Rivas-Perdomo E. Riesgo de muerte fetal por grupos etarios en una clínica universitaria en Cartagena, Colombia Rev. Cienc. Biomed. 2013;4(2):297-301.

18. Norwitz E; Edusa V; Shin Park J. Maternal physiology and complications of multiple pregnancy. Semin Perinatol 2005;29:338-348. 